\title{
ON THE GLOBAL EXISTENCE OF SOLUTIONS TO QUASILINEAR PARABOLIC EQUATIONS WITH HOMOGENEOUS NEUMANN BOUNDARY CONDITIONS
}

\author{
ZHAOYANG YIN \\ Department of Mathematics, Zhongshan University, 510275 Guangzhou, China* \\ Department of Mathematics, Lund University, PO Box 118, S-22100 Lund, Sweden ${ }^{\dagger}$
}

(Received 9 April, 2003; accepted 10 February, 2005)

\begin{abstract}
We prove under quite general assumptions the global existence of classical solutions for quasilinear parabolic equations in bounded domains with homogeneous Neumann boundary conditions.
\end{abstract}

2000 Mathematics Subject Classification. 35K55.

1. Introduction. Consider the following quasilinear parabolic problem

$$
\begin{cases}u_{t}-\operatorname{div}(a(t, x, u) \nabla u)=f(t, x, u, \nabla u), & t>0, x \in \Omega, \\ \frac{\partial u}{\partial v}(t, x)=0, & t>0, x \in \partial \Omega \\ u(0, x)=\varphi(x), & x \in \bar{\Omega},\end{cases}
$$

where $\Omega$ is a bounded domain in $\mathbb{R}^{n}$ with a $C^{2}$-boundary $\partial \Omega$, $v$ is the outer normal on $\partial \Omega$, and $\frac{\partial}{\partial v}$ stands for the derivative of $u$ in the direction of $v$.

Many authors have studied the problem (1) (see $[\mathbf{1}, \mathbf{2}, \mathbf{6}]$ and citations therein) by discussing the existence and uniqueness of local solutions, the global existence of solutions, blow-up behavior of solutions etc. Due to the difficulty produced by nonlinear part $f$, these problems for (1) still need to be investigated.

Our aim is to prove the global existence of classical solutions for the problem (1). Our method is not only based on maximum principles, but also relies on recent results for the time evolution of the extrema of a function [2,3] and comparison theorems [4]. This method permits us to obtain a new result for (1) under quite general assumptions on the nonlinearity. The result we obtain improves the recent result in [2].

Throughout this paper, we assume that

$$
a \in C^{2}\left(\mathbb{R}_{+} \times \bar{\Omega} \times \mathbb{R}, \mathbb{R}^{n \times n}\right), \quad f \in C^{1}\left(\mathbb{R}_{+} \times \bar{\Omega} \times \mathbb{R} \times \mathbb{R}^{n}, \mathbb{R}\right),
$$

and

$$
\langle a(t, x, w) \eta, \eta\rangle \geq c|\eta|^{2}, \quad(t, x, w) \in \mathbb{R}_{+} \times \bar{\Omega} \times \mathbb{R}, \eta \in \mathbb{R}^{n}
$$

\footnotetext{
*E-mail: mcsyzy@zsu.edu.cn

†E-mail: yin@maths.1th.se
} 
where $\mathbb{R}_{+}=[0, \infty)$ and $c>0$. Fix $p>n$. It is known from the classical parabolic theory $[\mathbf{1 , 6 ]}$ that, given initial data

$$
\varphi \in W=\left\{\phi: \phi \in W^{s, p}(\Omega),\left.\frac{\partial \phi}{\partial v}\right|_{\partial \Omega}=0\right\}, \quad s \in\left[1, \min \left\{1+\frac{1}{p}, 2-\frac{n}{p}\right\}\right),
$$

there exists some maximal $T=T(\varphi)>0$ and a unique solution

$$
u \in C^{1}((0, T) ; C(\bar{\Omega})) \cap C\left((0, T) ; C^{2}(\bar{\Omega})\right) \cap C([0, T) ; W) .
$$

Moreover, if $T<\infty$, one has

$$
\limsup _{t \uparrow T}\|u(t, \cdot)\|_{L^{\infty}(\Omega)}=\infty
$$

if the nonlinearity $f$ satisfies for $(t, x, w, \eta) \in \mathbb{R}_{+} \times \bar{\Omega} \times \mathbb{R} \times \mathbb{R}^{n}$ the growth condition

$$
|f(t, x, w, \eta)| \leq h(t, w)\left(1+|\eta|^{2}\right)
$$

with some $h \in C\left(\mathbb{R}_{+} \times \mathbb{R}, \mathbb{R}_{+}\right)$.

2. Main results. We prove that under certain conditions on the nonlinear part $f$, the solutions to (1) are global in time for any initial data $\varphi \in W$.

We first recall the notation of maximal and minimal solutions for ordinary differential equations. Let $w \in C\left(\mathbb{R}_{+} \times \mathbb{R}, \mathbb{R}\right)$ be given.

A solution $r \in C^{1}\left(\left[0, t_{0}\right), \mathbb{R}\right)$ with $t_{0}>0$, of the scalar differential equation $z^{\prime}=w(t, z(t))$ is said to be a maximal solution if for every solution $z$ of the equation on $\left[0, t_{0}\right)$ with $z(0)=r(0)$, the inequality

$$
z(t) \leq r(t), \quad t \in\left[0, t_{0}\right)
$$

holds. A minimal solution is defined similarly by reversing the above inequality. It is known (see e.g. [7]) that for every $w \in C\left(\mathbb{R}_{+} \times \mathbb{R}, \mathbb{R}\right)$ and every $z_{0} \in \mathbb{R}$, there exists some $t_{0}=t_{0}\left(z_{0}\right)>0$ such that there exist unique maximal and minimal solutions of the cauchy problem

$$
z^{\prime}=w(t, z(t)), \quad z(0)=z_{0},
$$

defined on $\left[0, t_{0}\right)$.

We will need the following three useful lemmas in the sequel.

Lemma 1 [4]. Let $w(t, z) \in C\left(\mathbb{R}_{+} \times \mathbb{R}, \mathbb{R}\right)$ and let $r(t)$ be the maximal solution of the ordinary differential equation

$$
z^{\prime}(t)=w(t, z), \quad z(0)=z_{0},
$$

defined on some interval $[0, T]$ with $T>0$. If $q(t):[0, T] \rightarrow \mathbb{R}$ is absolutely continuous and satisfies

$$
q^{\prime}(t) \leq w(t, q(t)), \quad \text { a.e. on }[0, T],
$$

with $q(0) \leq z_{0}=r(0)$, then $q(t) \leq r(t), t \in[0, T]$. 
Lemma 2 [4]. Let $w(t, z) \in C\left(\mathbb{R}_{+} \times \mathbb{R}, \mathbb{R}\right)$ and let $\rho(t)$ be the minimal solution of the ordinary differential equation

$$
z^{\prime}(t)=w(t, z), \quad z(0)=z_{0},
$$

defined on some interval $[0, T]$ with $T>0$. If $q(t):[0, T] \rightarrow \mathbb{R}$ is absolutely continuous and satisfies

$$
q^{\prime}(t) \geq w(t, q(t)), \quad \text { a.e. on }[0, T],
$$

with $q(0) \geq z_{0}=\rho(0)$, then $q(t) \geq \rho(t), t \in[0, T]$.

LEMMA 3 [2]. Let $T>0$ and $u \in W^{1,1}((0, T) ; C(\bar{\Omega}))$, where $\Omega$ is a bounded domain in $\mathbb{R}^{n}$. Then for every $t \in(0, T)$ there exists at least one pair of points $\xi(t), \zeta(t) \in \bar{\Omega}$ with

$$
m(t):=\min _{x \in \bar{\Omega}}[u(t, x)]=u(t, \xi(t)), \quad M(t):=\max _{x \in \bar{\Omega}}[u(t, x)]=u(t, \zeta(t)),
$$

and the functions $m(t), M(t)$ are absolutely continuous on $(0, T)$ with

$$
\frac{d m}{d t}(t)=u_{t}(t, \xi(t)) \quad \text { and } \quad \frac{d M}{d t}(t)=u_{t}(t, \zeta(t)) \quad \text { a.e. on }(0, T) .
$$

We now present the main results of the paper.

THEOREM 1. Assume that $f$ satisfies (2) and the condition

$$
w_{1}(t, u) \leq f(t, x, u, 0) \leq w_{2}(t, u)
$$

for some $w_{1}, w_{2} \in C\left(\mathbb{R}_{+} \times \mathbb{R}, \mathbb{R}\right)$. If for any initial data $\varphi \in W$ such that the minimal solution $\rho(t)$ of the scalar ordinary differential equation

$$
z_{t}=w_{1}(t, z), \quad z(0)=m(0)=\min _{x \in \bar{\Omega}}\{\varphi(x)\}
$$

exists globally in time and the maximal solution $r(t)$ of the scalar ordinary differential equation

$$
z_{t}=w_{2}(t, z), \quad z(0)=M(0)=\max _{x \in \bar{\Omega}}\{\varphi(x)\}
$$

exists globally in time, then the corresponding unique classical solution to (1) is defined globally in time.

Proof. Let $u$ be the classical solution of (1) with the initial data $\varphi$ and let $T>0$ be the maximal existence time of $u$. Set

$$
m(t):=\min _{x \in \bar{\Omega}}[u(t, x)], \quad M(t):=\max _{x \in \bar{\Omega}}[u(t, x)] .
$$

By Lemma 3, we know that $M(t)$ is absolutely continuous on $(0, T)$. Next, we prove that

$$
M^{\prime}(t) \leq w_{2}(t, M(t)) \quad \text { a.e. on }(0, T)
$$


Let $\zeta(t)$ be the point where $M(t)$ is attained. If $\zeta(t) \in \Omega$, then it follows that (see [5]) $\nabla u(t, \zeta(t))=0$ and

$$
\sum_{i, j=1}^{n} a_{i j}(t, \zeta(t), u(t, \zeta(t))) \frac{\partial^{2} u(t, \zeta(t))}{\partial x_{i} \partial x_{j}} \leq 0
$$

In view of the growth conditions of $f$, applying the above lemma and using the equation (1), we get

$$
\begin{aligned}
\frac{d M(t)}{d t} & =u_{t}(t, \zeta(t)) \\
& =\sum_{i, j=1}^{n} a_{i j}(t, \zeta(t), u(t, \zeta(t))) \frac{\partial^{2} u(t, \zeta(t))}{\partial x_{i} \partial x_{j}}+f(t, \zeta(t), u(t, \zeta(t)), 0) \\
& \leq w_{2}(t, M(t)) .
\end{aligned}
$$

Thus, we find that (3) holds true for $\zeta(t) \in \Omega$.

If $\zeta(t) \in \partial \Omega$, we construct a ball $B(\zeta(t), r)$ having center at $\zeta(t)$ and radius $r>0$. Let us consider the uniformly elliptic operator

$$
L u=\operatorname{div}(a(t, x, u) \nabla u)=\sum_{i, j=1}^{n} a_{i j} \frac{\partial^{2} u}{\partial x_{i} \partial x_{j}}+\sum_{j=1}^{n}\left(\sum_{i=1}^{n} \frac{\partial a_{i, j}}{\partial x_{i}}\right) \frac{\partial u}{\partial x_{j}}
$$

in $B(\zeta(t), r) \cap \Omega$. Since $M(t)$ is the maximum of $u(t, x)$ in $B(\zeta(t), r) \cap \Omega$ and $\frac{\partial u}{\partial v}(t, \zeta(t))=$ 0 , by the strong maximum principle (see [8]), we know that it is impossible to have $L u(t, x) \geq 0$ for all $x \in B(\zeta(t), r) \cap \Omega$ unless $u \equiv M(t)$ in $B(\zeta(t), r) \cap \Omega$.

If $u \equiv M(t)$ in $B(\zeta(t), r) \cap \Omega$, then we can choose $\zeta^{1}(t) \in \Omega$ instead of $\zeta(t)$, and we obtain the inequality (3).

Assume now $u$ is not constant in $B(\zeta(t), r) \cap \Omega$. Then there exists $x_{r} \in B(\zeta(t), r) \cap \Omega$ such that $L u\left(t, x_{r}\right)<0$. Therefore $L u(t, \zeta(t)) \leq 0$, since $r$ can be made arbitrarily small and $u(t, \cdot) \in C^{2}(\bar{\Omega})$. Next, let $\left\{\tau_{1}, \ldots, \tau_{n-1}\right\}$ be a basis of the tangent plane to $\partial \Omega$ at $\zeta(t)$. Using local coordinates and the fact that $\zeta(t)$ is a maximum point of $u$, it follows that

$$
\left\langle\nabla u(t, \zeta(t)), \tau_{j}\right\rangle_{\mathbb{R}^{n}}=0 \quad \text { for } \quad j=1, \ldots, n-1
$$

Note furthermore that $\partial_{\nu} u(t, \zeta(t))=\langle\nabla u(t, \zeta(t)), v(\zeta(t))\rangle_{\mathbb{R}^{n}}=0$ by the boundary condition in (1). We find that $\nabla u(t, \zeta(t))=0$ since

$$
\left\{\tau_{1}, \ldots, \tau_{n-1}, v(\zeta(t))\right\}
$$

forms a basis of $\mathbb{R}^{n}$. As in the case $\zeta(t) \in \Omega$, we also obtain the inequality (3) if $\zeta(t) \in \partial \Omega$.

Similarly, we can prove that

$$
m^{\prime}(t) \geq w_{1}(t, m(t)) \quad \text { a.e. on }(0, T)
$$


Provided $T<\infty$, it follows that $\limsup _{t \rightarrow T}\|u(t, \cdot)\|_{C(\bar{\Omega})}=\infty$. Thus, we obtain a sequence $\left\{t_{k}\right\} \subset(0, T)$ converging to $T$ such that

$$
\lim _{t_{k} \rightarrow T} M\left(t_{k}\right)=\infty \quad \text { or } \quad \lim _{t_{k} \rightarrow T} m\left(t_{k}\right)=-\infty .
$$

Suppose first that $\lim _{t_{k} \rightarrow T} M\left(t_{k}\right)=\infty$.

By the hypothesis of the theorem, we have that the maximal solution $r(t)$ of the scalar ordinary differential equation

$$
z_{t}=w_{2}(t, z), \quad z(0)=M(0)=\max _{x \in \bar{\Omega}}\{\varphi(x)\}
$$

exists globally in time. Applying Lemma 1, in view of (3), we obtain

$$
M(t) \leq r(t), \quad t \in \mathbb{R}_{+} .
$$

This contradicts the fact that $\lim _{t_{k} \rightarrow T} M\left(t_{k}\right)=\infty$.

Similarly, if $\lim _{t_{k} \rightarrow T} m\left(t_{k}\right)=-\infty$, by the hypothesis of the theorem, we have that the minimal solution $\rho(t)$ of the scalar ordinary differential equation

$$
z_{t}=w_{1}(t, z), \quad z(0)=m(0)=\min _{x \in \bar{\Omega}}\{\varphi(x)\}
$$

exists globally in time. Applying Lemma 2, in view of (4), we obtain

$$
m(t) \geq \rho(t), \quad t \in \mathbb{R}_{+} .
$$

This contradicts the fact that $\lim _{t_{k} \rightarrow T} m\left(t_{k}\right)=-\infty$. Therefore, $T=\infty$ and the solution $u$ exists globally in time. This completes the proof of the theorem.

As a particular case of Theorem 1, we obtain the following corollary.

COROLlary 1. Assume that $f$ satisfies (2) and the growth condition

$$
h(t, w) \leq \theta(t) \mu(w)
$$

with some $\theta \in C\left(\mathbb{R}_{+}, \mathbb{R}_{+}\right)$and $\mu \in C(\mathbb{R},(0, \infty))$.

(i) If

$$
\int_{0}^{\infty} \frac{d s}{\mu(s)}=\int_{-\infty}^{0} \frac{d s}{\mu(s)}=\infty,
$$

then for any initial data $\varphi \in W$ the corresponding unique classical solution to (1.1) is defined globally in time.

(ii) If an initial data $\varphi \in W$ satisfies

$$
\int_{0}^{\infty} \theta(s) d s<\min \left\{\int_{-\infty}^{m(0)} \frac{d s}{\mu(s)}, \int_{M(0)}^{\infty} \frac{d s}{\mu(s)}\right\},
$$

where $m(0)=\min _{x \in \bar{\Omega}}\{\varphi(x)\}$ and $M(0)=\max _{x \in \bar{\Omega}}\{\varphi(x)\}$, then the corresponding unique classical solution to (1) is defined globally in time. 
Proof. Let $u$ be the classical solution of (1) with the initial data $\varphi$ and let $T>0$ be the maximal existence time of $u$. Set

$$
m(t):=\min _{x \in \bar{\Omega}}[u(t, x)], \quad M(t):=\max _{x \in \bar{\Omega}}[u(t, x)] .
$$

By Lemma 3, we know that $M(t)$ and $m(t)$ are absolutely continuous on $(0, T)$. Using the growth condition of the corollary and following the similar proof on the inequalities (3) and (4), we can prove

$$
\frac{M^{\prime}(t)}{\mu(M(t))} \leq \theta(t) \quad \text { a.e. on }(0, T)
$$

and

$$
-\frac{m^{\prime}(t)}{\mu(m(t))} \leq \theta(t) \quad \text { a.e. on }(0, T)
$$

Provided $T<\infty$, it follows that $\limsup _{t \rightarrow T}\|u(t, \cdot)\|_{C(\bar{\Omega})}=\infty$. Thus, we obtain a sequence $\left\{t_{k}\right\} \subset(0, T)$ converging to $T$ such that

$$
\lim _{k \rightarrow \infty} M\left(t_{k}\right)=\infty \text { or } \quad \lim _{k \rightarrow \infty} m\left(t_{k}\right)=-\infty .
$$

(i) Suppose first that $\lim _{k \rightarrow \infty} M\left(t_{k}\right)=\infty$. Since $\theta(t) \in C\left((0, \infty), \mathbb{R}_{+}\right)$, we have that $\int_{t_{1}}^{T} \theta(s) d s=l<\infty$. Note that since $\int_{0}^{\infty} \frac{d s}{\mu(s)}=\infty$ and $\mu(s) \in C(\mathbb{R},(0, \infty))$, we have that there is a $n \in N$ such that

$$
\int_{M\left(t_{1}\right)}^{M\left(t_{n}\right)} \frac{d s}{\mu(s)}>l
$$

Thus, by (5), we have that

$$
l<\int_{M\left(t_{1}\right)}^{M\left(t_{n}\right)} \frac{d s}{\mu(s)}=\int_{t_{1}}^{t_{n}} \frac{M^{\prime}(t)}{\mu(M(t)} d t \leq \int_{t_{1}}^{T} \theta(s) d s=l .
$$

This leads to a contradiction. Similarly, if $\lim _{k \rightarrow \infty} m\left(t_{k}\right)=-\infty$, using (6), we also obtain a contradiction. Therefore, $T=\infty$ and the solution $u$ exists globally in time. This proves part (i) of the theorem.

(ii) Suppose first that $\lim _{k \rightarrow \infty} m\left(t_{k}\right)=-\infty$. Since $\int_{-\infty}^{m(0)} \frac{d s}{\mu(s)}>\int_{0}^{\infty} \theta(s) d s$, there is a $n \in N$ such that

$$
\int_{m\left(t_{n}\right)}^{m(0)} \frac{d s}{\mu(s)}>d=\int_{0}^{\infty} \theta(s) d s .
$$

Then (6) leads to the contradiction

$$
d<\int_{m\left(t_{n}\right)}^{m(0)} \frac{d s}{\mu(s)}=-\int_{0}^{t_{n}} \frac{m^{\prime}(t)}{\mu(m(t)} d t \leq \int_{0}^{\infty} \theta(s) d s=d .
$$

Similarly, if $\lim _{k \rightarrow \infty} M\left(t_{k}\right)=\infty$, using (5), we also obtain a contradiction. Therefore, $T=\infty$ and the solution $u$ exists globally in time. This completes the proof of corollary. 
EXAMPLE 1. Consider the problem (1) with

$$
|f(t, x, w, \nabla w)| \leq \frac{1}{(1+t)^{2}}\left(1+w^{2}\right)\left(1+|\nabla w|^{2}\right) .
$$

Take $\theta(t)=\frac{1}{2(1+t)^{2}}$ and $\mu(s)=1+s^{2}$, a straightforward computation shows that

$$
\int_{0}^{\infty} \theta(t) d t=\frac{1}{2}<\frac{\pi}{6}=\min \left\{\int_{\sqrt{3}}^{\infty} \frac{d s}{\mu(s)}, \int_{-\infty}^{-\sqrt{3}} \frac{d s}{\mu(s)}\right\} .
$$

Applying Corollary 1, we get that the solutions of (1) are global for any initial data satisfying $|\varphi(x)| \leq \sqrt{3}$ on $\bar{\Omega}$ if (7) holds. Observe that the recent results in [2] are not applicable here.

EXAMPLE 2. Consider the problem (1) with

$$
f(t, x, u, \nabla u)=t\left(u-u^{7}\right)\left(1+|\nabla u|^{2}\right) .
$$

Take $w_{1}(t, u)=w_{2}(t, u)=t\left(u-u^{7}\right)$. We have that the solutions of the scalar ordinary differential equation

$$
z_{t}=t\left(z-z^{7}\right), \quad z(0)=z_{0} .
$$

are unique as long as they exist in time. Therefore, the maximal solution of (9) is the unique solution of (9).

Next, we shall show that the unique solution of (9) with $z_{0}=M(0)=\max _{x \in \bar{\Omega}}\{\varphi(x)\}$ exists globally in time.

Multiplying equation (9) by $z(t)$, we obtain

$$
\frac{1}{2}\left(z^{2}(t)\right)^{\prime}=t\left(z^{2}-z^{8}\right) \leq t z^{2} .
$$

By means of the Gronwall inequality, we get

$$
z^{2}(t) \leq z^{2}(0) e^{t^{2}}, \quad t \in \mathbb{R}_{+} .
$$

Thus, we have

$$
|z(t)| \leq M(0) e^{\frac{t^{2}}{2}}, t \in \mathbb{R}_{+} .
$$

It follows that the unique solution of (9) with $z_{0}=M(0)=\max _{x \in \bar{\Omega}}\{\varphi(x)\}$ exists globally in time. Using an analagous argument, we can also prove that the minimal solution of (9) with $z_{0}=m(0)=\min _{x \in \bar{\Omega}}\{\varphi(x)\}$ exists globally in time.

Applying Theorem 1, we get that the solutions of (9) are global for any initial data. Observe that the recent results in [2] and [9] are not applicable here.

REMARK 1. Note that Theorem 2 in [2] and Corollary 1 are special cases of Theorem 1 with $w_{2}(t, u)=-w_{1}(t, u)=\gamma(u)$ and Theorem 1 with $w_{2}(t, u)=-w_{1}(t, u)=$ $\theta(t) \mu(u)$, respectively.

Using the Lyapunov functional and recent results on comparison theorems in Lemma 1 and Lemma 2, we can prove the following theorem. 
THEOREM 2. Assume that $f$ satisfies (2),

$$
f(t, 0,0)=0 \text { for all } t \in \mathbb{R}_{+},
$$

and there exists $V \in C^{1}\left(\mathbb{R}_{+} \times \mathbb{R}, \mathbb{R}\right)$ with

$$
\begin{gathered}
\lim _{|r| \rightarrow \infty} V(t, r)=\infty \text { for each fixed } t \in \mathbb{R}_{+}, \\
V_{r}(t, r) \geq 0 \quad \text { for all }(t, r) \in \mathbb{R}_{+} \times \mathbb{R}_{+}, \\
V_{r}(t, r) \leq 0 \quad \text { for all }(t, r) \in \mathbb{R}_{+} \times \mathbb{R}_{-},
\end{gathered}
$$

and such that for all $(t, r) \in \mathbb{R}_{+} \times \mathbb{R}$,

$$
V_{t}(t, r)+V_{r}(t, r) f(t, r, 0) \leq w(t, V(t, r)),
$$

for some $w \in C\left(\mathbb{R}_{+} \times \mathbb{R}, \mathbb{R}\right)$. If the initial data $\varphi \in W$ satisfies $\varphi \leq 0$ (resp. $\left.\varphi \geq 0\right)$ and is such that the maximal solution $r(t)$ of the scalar ordinary differential equation

$$
z_{t}=w(t, z), \quad z(0)=V(0),
$$

exists globally in time, where

$$
V(0)=V\left(0, \max _{x \in \bar{\Omega}} \varphi(x)\right)\left(\text { resp. } V(0)=V\left(0, \min _{x \in \bar{\Omega}} \varphi(x)\right) .\right.
$$

Then the corresponding unique classical solution to (1) is defined globally in time.

Proof. Let $u$ be the classical solution of (1) with the initial data $\varphi$ and let $T>0$ be the maximal existence time of $u$. Set

$$
m(t):=\min _{x \in \bar{\Omega}}[u(t, x)], \quad M(t):=\max _{x \in \bar{\Omega}}[u(t, x)] .
$$

By Lemma 3, we know that $M(t)$ and $m(t)$ are absolutely continuous on $(0, T)$. Following the similar proof on the inequalities (3) and (4), we can prove

$$
M^{\prime}(t) \leq f(t, M(t), 0) \quad \text { a.e. on }(0, T)
$$

and

$$
m^{\prime}(t) \geq f(t, m(t), 0) \quad \text { a.e. on }(0, T)
$$

Consider the ordinary differential system

$$
\frac{d u}{d t}=f(t, u, 0), \quad u(0)=0 .
$$

Note that $f \in C^{1}$ and (10) holds, so that $u(t) \equiv 0$ is the unique solution of (17). Thus, $u(t) \equiv 0$ is also the maximal solution of (17).

If the initial data $\varphi \leq 0$, then $M(0) \leq 0$. By Lemma 1 , we obtain that

$$
M(t) \leq 0 \quad t \in[0, T) .
$$

From the above relation, we infer that

$$
m(t) \leq M(t) \leq 0 \quad t \in[0, T) .
$$


Applying the inequalities (16) and (18), in view of hypotheses (13) and (14), we obtain

$$
\frac{d}{d t} V(t, m(t)) \leq w(t, V(t, m(t))) \quad \text { a.e. on }(0, T) .
$$

Provided $T<\infty$, it follows that

$$
\limsup _{t \rightarrow T}\|u(t, \cdot)\|_{C(\bar{\Omega})}=\infty .
$$

Thus, in view of (18), we obtain a sequence $\left\{t_{k}\right\} \subset(0, T)$ converging to $T$ such that

$$
\lim _{k \rightarrow \infty} m\left(t_{k}\right)=-\infty
$$

In view of (11), we have that $\lim _{k \rightarrow \infty} V\left(t_{k}, m\left(t_{k}\right)\right)=\infty$.

By the hypothesis of the theorem, we have that the maximal solution $r(t)$ of the scalar ordinary differential equation

$$
z_{t}=w(t, z), \quad z(0)=V(0, m(0))=V\left(0, \min _{x \in \bar{\Omega}} \varphi(x)\right),
$$

exists globally in time. Applying Lemma 1, in view of (19), we obtain

$$
V(t, m(t)) \leq r(t), \quad t \in \mathbb{R}_{+} .
$$

This contradicts $\lim _{t_{k} \rightarrow T} V\left(t_{k}, m\left(t_{k}\right)\right)=\infty$.

If the initial data $\varphi \geq 0$, then $m(0) \geq 0$. Note that $u(t) \equiv 0$ is also the minimal solution of (17). By Lemma 2, we obtain

$$
m(t) \geq 0, \quad t \in[0, T) .
$$

From the above relation, we deduce that

$$
M(t) \geq m(t) \geq 0, \quad t \in[0, T) .
$$

Applying the inequalities (15) and (20), in view of hypotheses (12) and (14), we obtain

$$
\frac{d}{d t} V(t, M(t)) \leq w(t, V(t, M(t))) \quad \text { a.e. on }(0, T)
$$

Provided $T<\infty$, it follows that

$$
\limsup _{t \rightarrow T}\|u(t, \cdot)\|_{C(\bar{\Omega})}=\infty .
$$

Thus, in view of (20), we obtain a sequence $\left\{t_{k}\right\} \subset(0, T)$ converging to $T$ such that

$$
\lim _{k \rightarrow \infty} M\left(t_{k}\right)=\infty
$$

By the hypothesis of the theorem, we have that the maximal solution $r(t)$ of the scalar ordinary differential equation

$$
z_{t}=w(t, z), \quad z(0)=V(0, M(0))=V\left(0, \max _{x \in \bar{\Omega}} \varphi(x)\right),
$$


exists globally in time. Applying Lemma 1, in view of (21), we obtain

$$
V(t, M(t)) \leq r(t), \quad t \in \mathbb{R}_{+} .
$$

This contradicts $\lim _{t_{k} \rightarrow T} V\left(t_{k}, M\left(t_{k}\right)\right)=\infty$. Therefore, $T=\infty$ and the solution $u$ exists globally in time. This completes the proof of the theorem.

As a particular case of Theorem 2, we have the following corollary.

COROllary 2. Assume that $f$ satisfies (2),

$$
f(t, 0,0)=0, \quad \text { for all } t \in \mathbb{R}_{+},
$$

and there exists $V \in C^{1}\left(\mathbb{R}_{+} \times \mathbb{R}, \mathbb{R}_{+}\right)$with

$$
\begin{gathered}
\lim _{|r| \rightarrow \infty} V(t, r)=\infty \text { for each fixed } t \in \mathbb{R}_{+}, \\
V_{r}(t, r) \geq 0 \quad \text { for all }(t, r) \in \mathbb{R}_{+} \times \mathbb{R}_{+}, \\
V_{r}(t, r) \leq 0 \quad \text { for all }(t, r) \in \mathbb{R}_{+} \times \mathbb{R}_{-},
\end{gathered}
$$

and such that for all $(t, r) \in \mathbb{R}_{+} \times \mathbb{R}$,

$$
V_{t}(t, r)+V_{r}(t, r) f(t, r, 0) \leq \theta(t) \mu(V(t, r)),
$$

for some $\theta \in C\left(\mathbb{R}_{+}, \mathbb{R}_{+}\right), \mu \in C\left(\mathbb{R}_{+},(0, \infty)\right)$. If the initial data $\varphi \in W$ satisfies $\varphi \leq 0$ (resp. $\varphi \geq 0$ ) and for all $T>0$

$$
\int_{0}^{T} \theta(t) d t<\int_{V(0)}^{\infty} \frac{d s}{\mu(s)},
$$

where

$$
V(0)=V\left(0, \max _{x \in \bar{\Omega}} \varphi(x)\right)\left(\operatorname{resp} . V(0)=V\left(0, \min _{x \in \bar{\Omega}} \varphi(x)\right)\right)
$$

then the corresponding unique classical solution to (1) is defined globally in time.

Proof. Let $u$ be the classical solution of (1) with the initial data $\varphi$ and let $T>0$ be the maximal existence time of $u$. Set

$$
m(t):=\min _{x \in \bar{\Omega}}[u(t, x)], \quad M(t):=\max _{x \in \bar{\Omega}}[u(t, x)] .
$$

If the initial data $\varphi \leq 0$, then $M(0) \leq 0$. Using the hypothesis of the corollary and following the same proof of (18) and (19), we can obtain

$$
m(t) \leq M(t) \leq 0 \quad t \in[0, T)
$$

and

$$
\frac{d}{d t} V(t, m(t)) \leq \theta(t) \mu(V(t, m(t))) \quad \text { a.e. on }(0, T) .
$$

Provided $T<\infty$, it follows that

$$
\limsup _{t \rightarrow T}\|u(t, \cdot)\|_{C(\bar{\Omega})}=\infty .
$$


Thus, in view of (22), we obtain a sequence $\left\{t_{k}\right\} \subset(0, T)$ converging to $T$ such that

$$
\lim _{k \rightarrow \infty} m\left(t_{k}\right)=-\infty
$$

In view of the hypothesis of the corollary, we have that

$$
\lim _{k \rightarrow \infty} V\left(t_{k}, m\left(t_{k}\right)\right)=\infty .
$$

For simplicity, we denote $V(t, m(t))$ by $V(t)$. Since $\theta(t) \in C\left((0, \infty), \mathbb{R}_{+}\right)$, we have that $\int_{0}^{T} \theta(s) d s=l<\infty$. Note that since $\int_{V(0)}^{\infty} \frac{d s}{\mu(s)}>l$ and $\mu(s) \in C(\mathbb{R},(0, \infty))$, we have that there is a $n \in N$ such that

$$
\int_{V(0)}^{V\left(t_{n}\right)} \frac{d s}{\mu(s)}>l .
$$

Thus, by (23), we would have a contradiction

$$
l<\int_{V(0)}^{V\left(t_{n}\right)} \frac{d s}{\mu(s)}=\int_{0}^{t_{n}} \frac{d V(t)}{\mu(V(t, m(t)))} \leq \int_{0}^{T} \theta(s) d s=l .
$$

This forces $T=\infty$.

If the initial data $\varphi \geq 0$, then $m(0) \geq 0$. Using the hypothesis of the corollary and following the same proof of (20) and (21), we can obtain

$$
M(t) \geq m(t) \geq 0, \quad t \in[0, T)
$$

and

$$
\frac{d}{d t} V(t, M(t)) \leq \theta(t) \mu(V(t, M(t))) \quad \text { a.e. on }(0, T) .
$$

Provided $T<\infty$, it follows that

$$
\limsup _{t \rightarrow T}\|u(t, \cdot)\|_{C(\bar{\Omega})}=\infty .
$$

Thus, in view of (24), we obtain a sequence $\left\{t_{k}\right\} \subset(0, T)$ converging to $T$ such that

$$
\lim _{k \rightarrow \infty} M\left(t_{k}\right)=\infty
$$

Similarly, using (25), we also obtain a contradiction. Therefore, $T=\infty$ and the solution $u$ exists globally in time. This completes the proof of the corollary.

EXAMPLE 3. Consider the following reaction-diffusion equation

$$
\begin{cases}u_{t}-\Delta u=u^{3} \frac{\left(1+4|\nabla u|^{2}\right)}{2(1+t)^{2}}, & t>0, x \in \Omega, \\ \frac{\partial u}{\partial \nu}(t, x)=0, & t>0, x \in \partial \Omega, \\ u(0, x)=\varphi(x), & x \in \bar{\Omega},\end{cases}
$$

where $\Omega$ is a bounded domain in $\mathbb{R}^{n}$ with a $C^{2}$-boundary $\partial \Omega$.

Note that $f(t, r, 0)=\frac{r^{3}}{2(1+t)^{2}}$ and set

$$
V(t, r)=r^{2}, \quad(t, r) \in \mathbb{R}_{+} \times \mathbb{R} .
$$


A straightforward computation shows that

$$
V_{t}(t, r)+V_{r}(t, r) f(t, r, 0)=\frac{r^{4}}{(1+t)^{2}}=\frac{V^{2}}{(1+t)^{2}} .
$$

Set $\theta(t)=\frac{1}{(1+t)^{2}}$ and $\mu(s)=s^{2}$. Observe that

$$
\int_{1}^{\infty} \frac{d s}{\mu(s)}=\int_{1}^{\infty} \frac{1}{s^{2}} d s=1=\int_{0}^{\infty} \frac{1}{(1+t)^{2}} d t
$$

Thus, the hypotheses of Corollary 2 are clearly satisfied. Applying Corollary 2, we have that if the initial data $\varphi \leq 0$ satisfies

$$
V(0)=\left[\min _{x \in \bar{\Omega}} \varphi(x)\right]^{2} \leq 1
$$

or the initial data $\varphi \geq 0$ satisfies

$$
V(0)=\left[\max _{x \in \bar{\Omega}} \varphi(x)\right]^{2} \leq 1,
$$

then the corresponding solution to (26) exists globally in time. Observe that the recent results in $[2,4]$ are ineffective here.

REMARK 2. Note that Theorem 1 in [9] and Corollary 2 are special cases of Theorem 2 with $w(t, V(t, r))=\theta(t) \mu(V(t, r))$.

ACKNOWLEDGMENTS. This work was partially supported by the NNSF of China, the SRF for ROCS, SEM, the NSF of Guangdong Province, and the Foundation of Zhongshan University Advanced Research Center and was performed as a Visiting Researcher at Lund University. The author is very pleased to acknowledge the support and encouragement of Professor A. Constantin as this work has developed. The author thanks the referee for several useful suggestions and comments that improved considerably the initial version of this paper.

\section{REFERENCES}

1. H. Amann, Dynamic theory of quasilinear parabolic systems. III. Global existence, Math. Z. 202 (1989), 219-250.

2. A. Constantin and J. Escher, Global solutions for quasilinear parabolic problems, J. Evolution Equations 2 (2002), 97-111.

3. A. Constantin and J. Escher, Wave breaking for nonlinear nonlocal shallow water equations, Acta Mathematica 181 (1998), 229-243.

4. A. Constantin, J. Escher and Z. Yin, Global Solutions for Quasilinear Parabolic Systems, J. Differential Equations 197 (2004), 73-84.

5. D. Gilbarg and N. S. Trudinger, Elliptic partial differential equations of seconder order (Springer-Verlag, 1977).

6. A. Lunardi, Analytic semigroups and optimal regularity in parabolic equations (Birkhöuser Verlag, 1995). 1998)

7. B. G. Pachpatte, Inequalities for differential and integral equations (Academic Press,

8. M. H. Protter and H. F. Weinberger, Maximum principles in differential equations (Prentice-Hall, Inc. Englewood Cliffs, N.J. 1967).

9. Z. Yin, Global existence for quasilinear parabolic systems with homogeneous Neumann boundary conditions, Nonlinear Differential Equations and Applications, in press. 